\title{
Induction thermography - a non-destructive testing method
}

\author{
by B.Oswald-Tranta
}

\begin{abstract}
Institute of Automation, University of Leoben, Peter-Tunnerstr. 27, A-8700, Leoben, Austria,
\end{abstract} beate.oswald@unileoben.ac.at

\begin{abstract}
In the last years inductive thermography has been well established as a non-destructive testing technique, which can be excellently used for detecting failures in electrically conductive materials. A short inductive heating pulse $(0.1-1 \mathrm{~s})$ is applied to the workpiece, and due to the induced eddy currents Joule heating is generated inside the material. The surface temperature and its temporal evolution are recorded by an infrared camera. As defects disturb the eddy current distribution and the heat flow, they can be well recognised in the infrared images. Evaluating the whole infrared image sequence instead of only one single thermogram increases strongly the contrast and the signal-to-noise ratio. The technique can be used to detect surface cracks in metals as well as for detection of subsurface defects, but also for localizing failures, such as impact defects, in CFRP structures.
\end{abstract}

\section{Surface cracks in metals}

Surface cracks in metals can be well detected by inductive thermography, as the induced eddy current and the heat flow are deflected by the cracks. Therefore the surface temperature in the vicinity of a crack becomes inhomogeneous. Theoretical investigations, analytical models and finite element simulations are used to determine the optimal parameters for a measurement, as heating pulse duration and evaluation time [1]. If $\delta$, the penetration depth of the eddy current is significantly less than the crack depth, as it is the case for ferro-magnetic steel, then the crack is selectively heated and can be recognised due to increased temperature, see Fig.1a. On the other hand, if the penetration depth is larger or about the same size as the crack depth, as it is e.g. the case for titanium, then the surface temperature around the crack is less than at the sound, defect-free surface, see Fig.1b. These FEM simulations were carried out assuming long cracks, as e.g. rolling laps. If the crack is not perpendicular to the surface, but inclined, then the surface temperature distribution is asymmetric. In the case of ferro-magnetic steel with small eddy current penetration depth, in the region with small angle the heat is accumulated, see Fig.1c. In contrast, for titanium with large penetration depth this region is colder (Fig.1d).
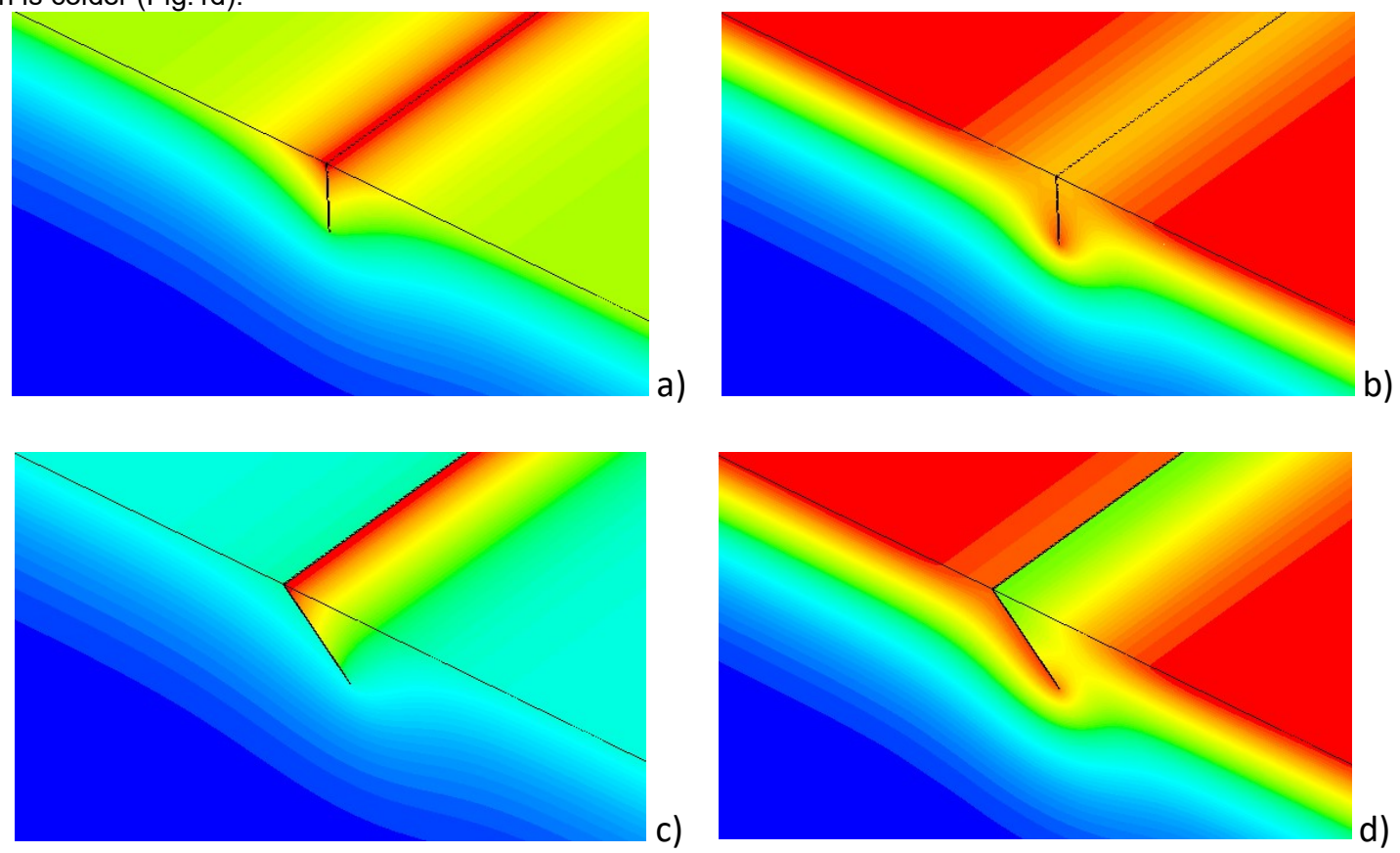

Fig. 1: FEM simulation results after $0.1 \mathrm{~s}$ inductive heating pulse. The crack depth is in each case $1 \mathrm{~mm}$, in figs. a and b the crack is perpendicular to the surface; and in the case of figs. $c$ and $d$ it is inclined by $45^{\circ}$. Figs.a and c show results simulated for ferro-magnetic steel, where the penetration depth is about $0.03 \mathrm{~mm}$, if the excitation frequency is $200 \mathrm{kHz}$. Figs. $b$ and $d$ show simulation results for titanium, in this case the penetration depth is $0.8 \mathrm{~mm}$ for $200 \mathrm{kHz}$. 


\subsection{1/qirt.2019.004}

Experimental results show the behaviour as it is expected according to the simulations, see Fig.2. In this figure not temperature images, but phase images are depicted. It has been proven [1] that if the whole infrared sequence of the heating and cooling durations are evaluated by Fourier transformation, then effects, as inhomogeneous heating or inhomogeneous emissivity properties will be strongly reduced in the phase image, and the contrast and the signal-tonoise ratio becomes strongly increased.
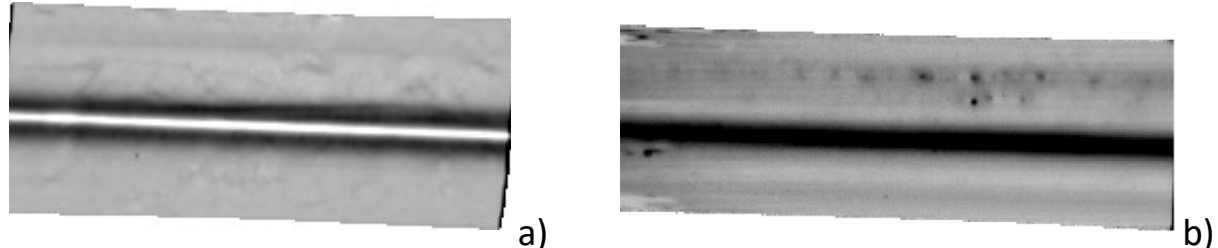

a)

Fig. 2: Phase images of $0.1 \mathrm{~s}$ heating pulse measurement; a: ferro-magnetic steel wire with $30 \mathrm{~mm}$ diameter; $b$ : titanium wire with $20 \mathrm{~mm}$ diameter. The cracks, typical rolling laps, are visible along the whole length of the samples.

Inductive thermography has the advantage that it is quick, contact-free and it can be fully automated. Different image processing techniques have been developed to recognise defects in the recorded infrared images and to distinguish between real failures and artifacts. The presentation gives an overview about the inductive thermography method and its application for several different types of workpieces.

\section{Inductive testing of CFRP}

Carbon fibers of reinforced plastic can also conduct electrical current, therefore it can be also heated by induced eddy currents. The geometry of the induction coil is 'mirrored' to the specimen [2]. If the coil has a circular form, then only fibers tangential to the coil are heated, resulting the pattern in Fig.3a. In contrast, a Helmholtz coil generates in its mid area a homogeneous magnetic field and the eddy current flows along fibers in one direction, see Fig.3b. As this pattern is more homogeneous, defects as e.g. impact damage (see Fig.3c) can be more easily recognized.
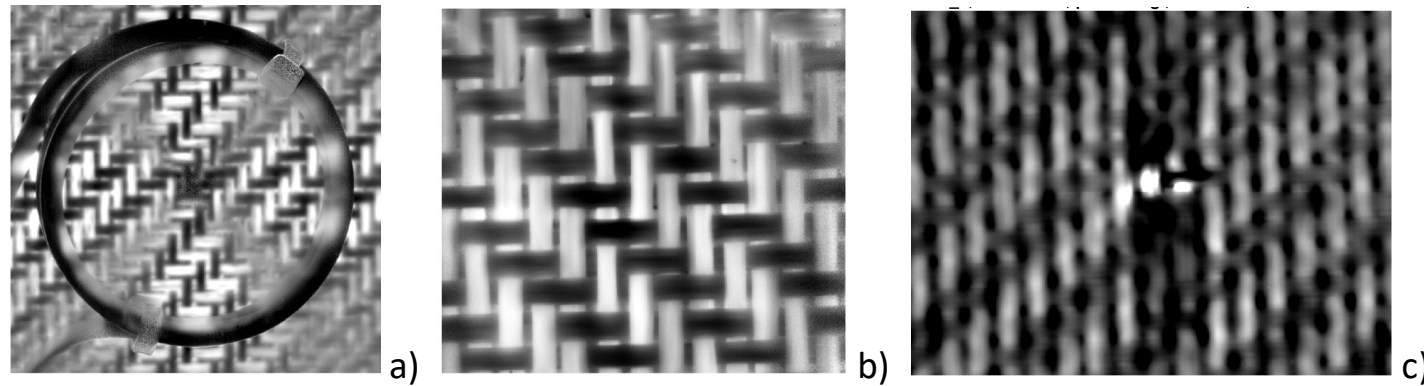

Fig. 3: Phase images of CFRPs after $1 \mathrm{~s}$ inductive heating; in figs. a and $b$ one ply CFRP fabric without resin matrix is shown, in fig. a it was heated by a circular induction coil placed above the fabric. In fig.b the fabric was placed in the mid region of a Helmholtz coil. In fig.c a CFRP structure containing three plies is depicted heated by a Helmholtz coil. In the middle of the image small impact damage can be recognised due to the higher phase values.

\section{REFERENCES}

[1] B.Oswald-Tranta, Induction Thermography for Surface Crack Detection and Depth Determination, Appl. Sci.8, 257; http://dx.doi.org/10.3390/app8020257 (2018)

[2] Miller AK, et al. The nature of induction heating in graphite-fibre, polymer-matrix composite materials, SAMPE Journal 1990; 26(4):37-54.

[3] Oswald-Tranta B., Tuschl C., Schledjewski R, „Flash and inductive thermography for CFRP inspection“, Proc. SPIE, Thermosense XLI, vol. 11004, 2019. 